\title{
Reliability analysis of flood embankments taking into account a stochastic distribution of hydraulic loading
}

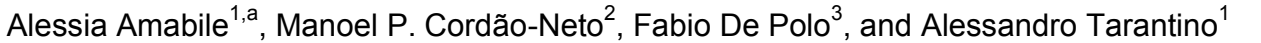 \\ ${ }^{1}$ University of Strathclyde, Civil and Environmental Engineering Department, Glasgow, UK \\ ${ }^{2}$ Universidade de Brasilia, Brasilia, Brazil \\ ${ }^{3}$ Hydraulic Department, Autonomous Province of Bolzano, Bolzano, Italy
}

\begin{abstract}
Flooding is a worldwide phenomenon. Over the last few decades the world has experienced a rising number of devastating flood events and the trend in such natural disasters is increasing. Furthermore, escalations in both the probability and magnitude of flood hazards are expected as a result of climate change. Flood defence embankments are one of the major flood defence measures and reliability assessment for these structures is therefore a very important process. Routine hydro-mechanical models for the stability of flood embankments are based on the assumptions of steady-state through-flow and zero pore-pressures above the phreatic surface, i.e. negative capillary pressure (suction) is ignored. Despite common belief, these assumptions may not always lead to conservative design. In addition, hydraulic loading is stochastic in nature and flood embankment stability should therefore be assessed in probabilistic terms. This cannot be accommodated by steady-state flow models. The paper presents an approach for reliability analysis of flood embankment taking into account the transient water through-flow. The factor of safety of the embankment is assessed in probabilistic terms based on a stochastic distribution for the hydraulic loading. Two different probabilistic approaches are tested to compare and validate the results.
\end{abstract}

\section{Introduction}

Current methods for assessing the stability of flood embankments are mostly deterministic. This means they handle hydro-mechanical loading as if it was precisely known; this is not the case when dealing with natural hazards such as floods, which are stochastic phenomena. The natural variability of soil properties or the scarce amounts of field and laboratory experimental data also add uncertainty to the characterisation of mechanical and hydraulic properties of materials.

Probabilistic approaches enable randomness and uncertainty in the loading and soil hydro-mechanical properties to be taken into account by defining them as random variables described by a probability distribution.

This paper focuses on the uncertainty of the hydraulic loading in the stability analysis of flood embankments. The hydraulic loading is represented by a hydrograph, i.e. the change of river level over time. The uncertainty in the hydraulic loading has been taken into account by:

- identifying a number of key variables to characterise the hydrograph;

- identifying the probability distribution function of each key variable;

- assessing the relative influence of each key variable on the result of the stability analysis.

The output of a deterministic stability analysis is a single value of factor of safety; if this value is greater than unity the embankment is considered to be stable. On

${ }^{\mathrm{a}}$ Corresponding author: alessia.amabile@strath.ac.uk the other hand, the output of a probabilistic analysis is the probability distribution of the factor of safety. Its mean and standard deviation can be used to calculate the probability of failure of the embankment, i.e. the probability that the factor of safety is lower than unity.

Two different probabilistic approaches, i.e. FOSM and Monte Carlo method, have been applied to assess the stability of an ideal embankment. An embankment in the North of Italy on the Adige River has been chosen as a reference.

\section{Probabilistic methods}

\subsection{First Order Second Moment method}

The First Order Second Moment (FOSM) method is based on a Taylor's series expansion of the performance function $Y=g\left[X_{1}, X_{2}, \ldots, X_{n}\right]$ around its mean value, where $X_{1}, \ldots, X_{n}$ are the input random variables. Only first order terms of the series are taken into account and only the first and second moment, i.e. mean and standard deviation $\sigma$, are considered, hence the name First Order Second Moment method. If the input variables are not correlated, the mean $E[Y]$ and the variance $\sigma^{2}[Y]$ of the performance function are given by Eq. (1) and Eq. (2) respectively:

$$
E[Y] \cong g\left(E\left[X_{1}\right], E\left[X_{2}\right], \ldots, E\left[X_{n}\right]\right)
$$




$$
\sigma^{2}[Y] \cong \sum_{1}^{n}\left(\frac{\partial g}{\partial X_{i}}\right)^{2} \sigma^{2}\left[X_{i}\right]
$$

If a probability distribution function is assumed for the performance function $Y$, its mean and standard deviation can be used to calculate the probability for any of its values. If input variables are not independent, correlation has to be taken into account for the calculation of the variance $\sigma^{2}[Y]$. The assumption of the probability distribution function for the performance function is one of the limitations of the FOSM method.

The analytical calculation of the partial derivatives in Eq. (2) may be complex for many geotechnical problems; they can be estimated numerically by computing the performance function at two different points. A common practice [1] is to select two points over a range of plus and minus one standard deviation with respect to the mean value of the random variable $X_{i}$, in order to capture the nonlinear behaviour of the function in a range of likely values.

One of the major advantages of FOSM method is that the terms $\left(\frac{\partial g}{\partial X_{i}}\right)^{2} \sigma^{2}\left[X_{i}\right]$ provide an immediate quantitative assessment of the influence of the variability of each input variable on the variance of the performance function.

\subsection{Monte Carlo method}

Monte Carlo method is very commonly used to obtain estimates of the solution of complex mathematical problems. It is a useful tool when the physical laws governing a certain process are known, but the problem cannot be solved analytically. This is frequently the case in geotechnical engineering, where closed-form solutions are not available for many common problems [2].

The Monte Carlo method is based on the repeated sampling of random numbers [3]. Each random number is associated to a value of the input variable $X_{i}$ via its probability distribution. The performance function is evaluated at different points, corresponding to different sets of random values of the input variables $X_{i}$. This process is repeated many times in order to cover all the possible range of outcomes. All the resulting values of the performance function are then aggregated to obtain an estimate of its probability distribution, from which the mean and standard deviation can be calculated.

The number of points at which the performance function $Y$ needs to be evaluated to have a good estimate of the probability distribution, i.e. the number of simulations to run, depends on the number of input variables. If the number of input variables increases the number of required simulations increases accordingly: the process becomes more and more time-consuming and computationally heavy.

\section{Hydrological loading}

Hydrological data used in this paper are referred to measurements taken in Bronzolo (BZ) on the Adige River, in the North of Italy, where a hydrometer is located. In this section river level variations have been consistently recorded for the past 30 years, thus allowing for the development of a probabilistic model for the hydraulic loading.

The dataset consists of:

- 82 records of annual maximum river level $h_{\text {peak }}$ from 1924 to 2005 ;

- 30 recorded hydrographs of all the flood events occurred between 1977 and 2014.

\subsection{The hydrograph}

In common approaches for embankment stability analysis, a steady-state flow is considered and the hydraulic loading is described by a single parameter, i.e. the maximum river level $h_{\text {peak }}$. In transient-state analysis, on the other hand, a time-dependent hydraulic loading has to be considered. This means that the flood event is not defined by the single value $h_{\text {peak }}$, but by a function describing the evolution in time of the river level, i.e. the hydrograph. In this case not only the peak, but also factors like duration or parameters controlling the shape of the hydrograph have to be taken into account.

Between 1977 and 2014, 30 flood events have been recorded by the hydrometer in Bronzolo. From the analysis of the recorded flood hydrographs an attempt has been made to develop a mathematical function able to describe a "typical" hydrograph in the section under consideration. The advantage of this approach is that a function makes the model flexible and easily adaptable to hydrographs recorded at different locations; moreover it can be conveniently reproduced and implemented in any software.

Four key variables have been selected and identified to describe a flood event:

- $\square h_{\text {peak }}$ : maximum river level reached during the flood event;

- $\square h_{\text {start }} / h_{\text {peak }}$ : ratio between the initial river level $h_{\text {start }}$, at the beginning of the flood event, and the maximum river level;

- $\square t_{\text {rise }}$ : time elapsed between the beginning of the flood event $\left(h_{\text {start }}\right)$ and the moment corresponding to the maximum river level $\left(h_{\text {peak }}\right)$;

- $\square \alpha$ : shape parameter controlling the falling limb of the hydrograph.

A representation of the hydrograph and the key variables is shown in Fig. (1).

The rising limb of the hydrograph, before the peak, can be well described by a linear function; the parameters governing this part are $t_{\text {rise }}, h_{\text {start }}$ and $h_{\text {peak }}$, as shown in Eq. (3). For the falling limb of the hydrograph $\left(t>t_{\text {rise }}\right)$ among different functions the power decay has shown the best fitting to the recorded hydrographs. The shape of the decreasing part of the hydrographs therefore depends not only on the shape parameter $\alpha$ but also on $t_{\text {rise }}$, as shown in Eq. (4).

$$
\begin{array}{ll}
h=h_{\text {start }}+\frac{h_{\text {peak }}-h_{\text {start }}}{t_{\text {rise }}} t & \left(t \leq t_{\text {rise }}\right) \\
h=h_{\text {peak }} *\left(\frac{t}{t_{\text {rise }}}\right)^{(-\alpha)} & \left(t>t_{\text {rise }}\right)
\end{array}
$$


For each recorded hydrograph the best-fitting values of $t_{\text {rise }}, h_{\text {start }}$ and $\alpha$ have been found by minimising the least square error between the recorded hydrograph and the one described by functions in Eq. (3) and Eq. (4).

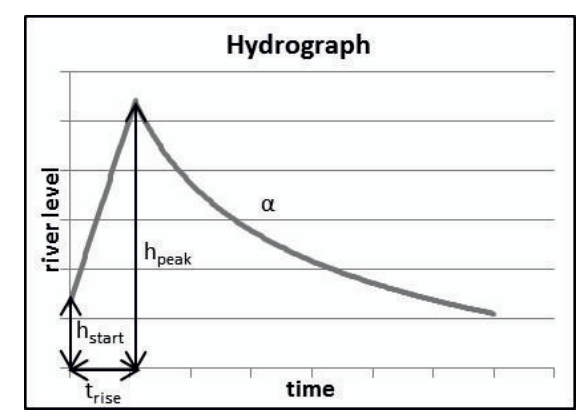

Figure 1. Representation of the hydrograph.

\subsection{Probabilistic modelling of floods}

After identifying a function to define the hydrograph the probabilistic model for the flood has been developed by identifying the probability distribution of each key variable, under the assumption that they are independent.

The probabilistic distribution of the peak river level of the hydrograph $h_{\text {peak }}$ has been assessed in the conventional way, i.e. deriving the cumulative probability distribution function from the annual maximum series. The empirical cumulative distribution function has been fitted with a Gumbel distribution, which is commonly employed in flood frequency analysis.

The empirical cumulative distribution functions for all the other parameters have been derived from the analysis of past flood events, not necessarily associated with the maximum annual river level. The empirical distribution functions have been fitted with a normal distribution. Results are reported in Fig. (2) for $h_{\text {peak }}$ and Table (1) for $h_{\text {start }} / h_{\text {peak }}, t_{\text {rise }}$ and $\alpha$.

Table 1. Mean and standard deviation of normal probability distribution function for the variables $h_{\text {start }} / h_{\text {peak }}, t_{\text {rise }}$ and $\alpha$.

\begin{tabular}{|c|c|c|}
\hline Variable & $\boldsymbol{\mu}$-Normal & $\boldsymbol{\sigma}$-Normal \\
\hline $\mathbf{h}_{\text {start }} / \mathbf{h}_{\text {peak }}$ & 0.613 & 0.094 \\
\hline $\mathbf{t}_{\text {rise }}[\mathbf{d}]$ & 1.077 & 0.371 \\
\hline $\boldsymbol{\alpha}$ & 0.278 & 0.095 \\
\hline
\end{tabular}

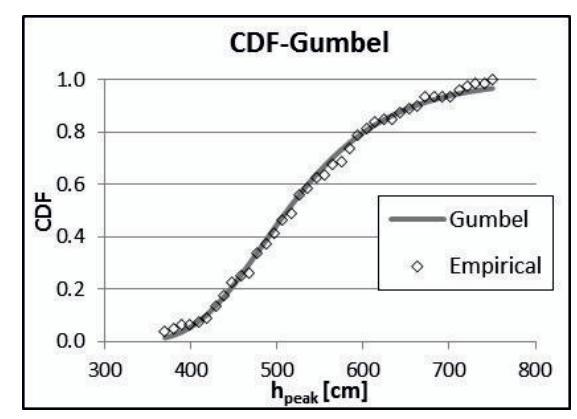

Figure 2. Empirical and Gumbel cumulative probability distribution function for the variable $h_{p e a k}$.

\section{The hydro-mechanical model}

\subsection{Geometry and materials}

An embankment in the North of Italy on the Adige River has been chosen as a reference. The embankment is about $8 \mathrm{~m}$ high from the riverbed and about $5.5 \mathrm{~m}$ high from the ground surface on the landside. The simplified geometry and soil profile of the cross-section is shown in Fig. (3).

The original soil profile has been simplified into two homogeneous layers, the first one $F$ including the foundation and the second one $E$ including the embankment and the shallow layer of agricultural soil on the landside.

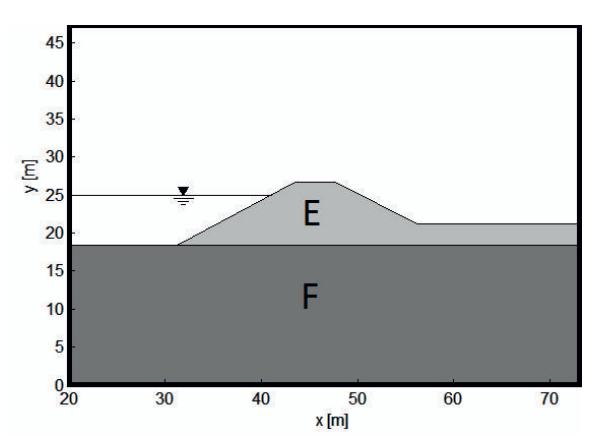

Figure 3. Geometry and soil profile of the embankment.

Van Genuchten model has been used to characterise the hydraulic behaviour of materials under unsaturated condition [4]. The equations for the effective saturation, the volumetric water content, and the relative hydraulic conductivity are defined in Eq. (5), Eq. (6) and Eq. (7) respectively:

$$
\begin{aligned}
& S_{e}=\frac{1}{\left(1+\left(\alpha_{V G} s\right)^{n}\right)^{m}} \\
& \theta=\theta_{\text {res }}+\left(\theta_{\text {sat }}-\theta_{\text {res }}\right) S_{e} \\
& k_{\text {rel }}=S_{e}^{-\lambda}\left(1-\left(1-S_{e}^{\frac{1}{m}}\right)^{m}\right)^{2}
\end{aligned}
$$

where

$$
\mathrm{m}=1-\frac{1}{\mathrm{n}}
$$

$s$ is the suction and $\theta_{\text {res }}$ and $\theta_{\text {sat }}$ are the residual volumetric water content and the saturated volumetric water content respectively.

Plots of water retention and relative hydraulic conductivity curves are shown in Fig. (4). The material in the foundation layer is always saturated; for this reason the value of the coefficient $\lambda$ has been chosen in order to have a drop of just one order of magnitude in the hydraulic conductivity. A small drop in the hydraulic conductivity makes the computation numerically easier without affecting the accuracy of results since this range of suction is never explored in the simulation.

For each material, the values of $\theta_{\text {res }}$ and $\theta_{\text {sat }}, \alpha, n$ and $\lambda$ are reported in Table (2), together with the mechanical properties and the values of saturated hydraulic conductivity. 


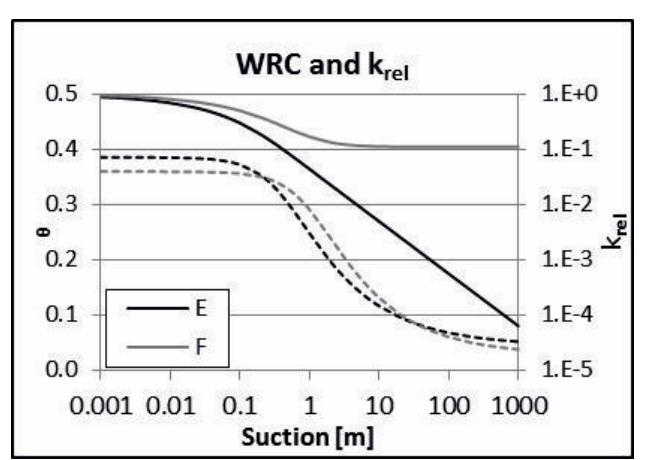

Figure 4. Water retention curve (continuous lines) and relative hydraulic conductivity curve (dashed lines) for the foundation layer (F) and the embankment/shallow layer on landside (E).

Table 2. Material properties for foundation layer $(\mathrm{F})$ and embankment and shallow layer on landside (E).

\begin{tabular}{|c|c|c|c|}
\hline \multicolumn{2}{|c|}{ Material properties } & F & E \\
\hline Friction angle & $\varphi^{\prime}\left[{ }^{0}\right]$ & 33 & 30 \\
\hline Cohesion & $\mathrm{c}^{\prime}[\mathrm{kPa}]$ & 0 & 0 \\
\hline Unit weight & $\begin{array}{c}\gamma \\
{\left[\mathrm{kN} / \mathrm{m}^{3}\right]}\end{array}$ & 20 & 20 \\
\hline $\begin{array}{c}\text { Sat. hydraulic } \\
\text { conductivity }\end{array}$ & $\mathrm{k}_{\mathrm{H}}[\mathrm{m} / \mathrm{s}]$ & 0.001 & 0.0005 \\
\hline Res. water content & $\theta_{\mathrm{res}}$ & 0.029 & 0.045 \\
\hline Sat. water content & $\theta_{\mathrm{sat}}$ & 0.360 & 0.386 \\
\hline \multirow{2}{*}{$\begin{array}{c}\text { Parameters for Van } \\
\text { Genuchten }\end{array}$} & $\alpha_{\mathrm{VG}}\left[\mathrm{m}^{-1}\right]$ & 1.0 & 2.5 \\
\cline { 2 - 5 } hydraulic functions & $\mathrm{n}_{\mathrm{VG}}$ & 1.50 & 1.48 \\
\cline { 2 - 4 } & $\lambda_{\mathrm{VG}}$ & 6 & 4.2 \\
\hline
\end{tabular}

\subsection{The seepage analysis}

A transient-state seepage analysis has been performed in order to evaluate the distribution of pore water pressure in the embankment during the flood event. The boundary condition on the riverside is given by the time-dependent hydraulic head represented by the hydrograph; the boundary condition on the slope of the embankment and the ground surface is a seepage face.

The initial condition in the transient-state seepage analysis has been obtained from a steady-state analysis associated with a river level equal to $h_{\text {start }}$, i.e. the initial river level for the flood event. For the steady-state analysis on the far end vertical boundary on the landside, $80 \mathrm{~m}$ away from the toe of the embankment, the ground water table has been assumed to be located at the interface between the two layers.

\subsection{The stability analysis}

For the stability analysis Bishop's simplified method has been used. This is one of the so called methods of slices, which discretise the soil mass by dividing it in slices and are commonly used when analysing stability of slopes in 2D. The soil is assumed to have rigid-perfectly plastic behaviour, i.e. that the soil does not show any deformation before failure; for this reason the solution of the problem is based only on equilibrium equations.
Bishop's simplified method is based on the assumptions of circular slip surface and zero inter-slice shear forces; it satisfies vertical force equilibrium for each slice and overall moment equilibrium about the centre of the circular slip surface. In order to solve the problem an iterative procedure is required.

\section{Application and results}

\subsection{FOSM}

In the application of the FOSM method to the stability analysis of the embankment taking into account the uncertainty in the hydrological loading, the input variable $X_{1}, \ldots, X_{n}$ are represented by the four key variables identified to describe the hydrograph $\left(h_{\text {peak }}, t_{\text {rise }}, h_{\text {start }} / h_{\text {peak }}\right.$ and $\alpha$ ) and the performance function $Y=g\left[X_{1}, X_{2}, \ldots, X_{n}\right]$ is represented by the factor of safety $F S$.

The mean and standard deviation of the factor of safety can be calculated as:

$$
\begin{aligned}
E[F S] \cong & g\left(E\left[h_{\text {peak }}\right], E\left[h_{\text {2start }}\right], E\left[t_{\text {rise }}\right], E[\alpha]\right) \\
\sigma^{2}[F S] \cong & \left(\frac{\partial g}{\partial h_{\text {peak }}}\right)^{2} \sigma^{2}\left[h_{\text {peak }}\right]+\left(\frac{\partial g}{\partial h_{\text {start }}}\right)^{2} \sigma^{2}\left[h_{\text {start }}\right]+ \\
& \left(\frac{\partial g}{\partial t_{\text {rise }}}\right)^{2} \sigma^{2}\left[t_{\text {rise }}\right]+\left(\frac{\partial g}{\partial \alpha}\right)^{2} \sigma^{2}[\alpha]
\end{aligned}
$$

The number of simulations required to apply the FOSM method is $1+2 n$, where $n$ is the number of input variables. In order to calculate the mean value of the factor of safety, the mean value of each input variable is considered ( 1 simulation). In order to calculate the variance of the factor of safety, for each input variables the factor of safety is calculated in two points, correspondent to plus and minus one standard deviation with respect to the mean value ( $2 n$ simulations). By keeping all variables except one equal to their mean values nine hydrographs have been produced by combining these values, as shown in Table (3) and Fig. (5).

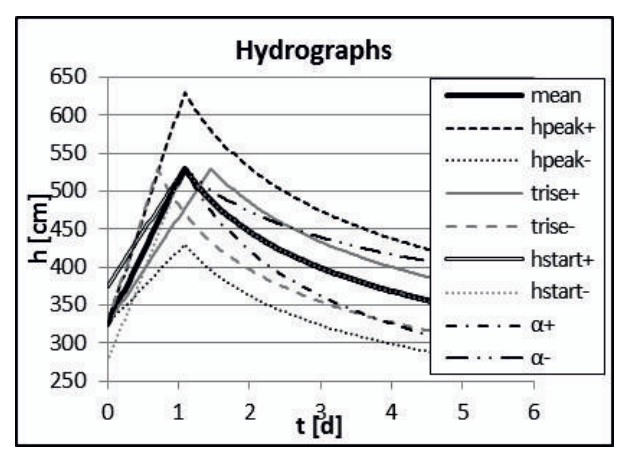

Figure 5. Hydrographs obtained from combinations of values of input variables for the application of FOSM method.

A normal probability distribution function is assumed for the factor of safety; the need to assume the distribution function for the performance function is one of the limitations of FOSM method. Another limitation in the application to stability analysis is the need to take into account a single slip surface. The critical slip surface 
obtained at the peak from the analysis with the mean values of all the input variables is considered.

Seepage analyses have been performed with the software SEEP/W and stability analyses have been performed with the software SLOPE/W. The mean value and standard deviation of the factor of safety have been calculated at different times and results are shown in Fig. (6). The minimum value of the mean of the factor of safety corresponds to the peak of the hydrograph.

Table 3. Combinations of values of input variables for the application of FOSM method.

\begin{tabular}{|c|c|c|c|c|}
\hline & $\mathbf{h}_{\text {peak }}[\mathbf{c m}]$ & $\mathbf{t}_{\text {rise }}[\mathbf{d}]$ & $\mathbf{h}_{\text {start }}[\mathbf{c m}]$ & $\boldsymbol{\alpha}$ \\
\hline mean & 530 & 1.083 & 325 & 0.278 \\
\hline $\mathrm{h}_{\text {peak }}+$ & $\underline{630}$ & 1.083 & 325 & 0.278 \\
\hline $\mathrm{h}_{\text {peak }}$ & $\underline{430}$ & 1.083 & 325 & 0.278 \\
\hline $\mathrm{t}_{\text {rise }}+$ & 530 & $\underline{1.458}$ & 325 & 0.278 \\
\hline $\mathrm{t}_{\text {rise }}{ }^{-}$ & 530 & $\underline{0.708}$ & 325 & 0.278 \\
\hline $\mathrm{h}_{\text {start }}+$ & 530 & 1.083 & $\underline{375}$ & 0.278 \\
\hline $\mathrm{h}_{\text {start }}{ }^{-}$ & 530 & 1.083 & $\underline{275}$ & 0.278 \\
\hline$\alpha+$ & 530 & 1.083 & 325 & $\underline{0.373}$ \\
\hline$\alpha-$ & 530 & 1.083 & 325 & $\underline{0.184}$ \\
\hline
\end{tabular}

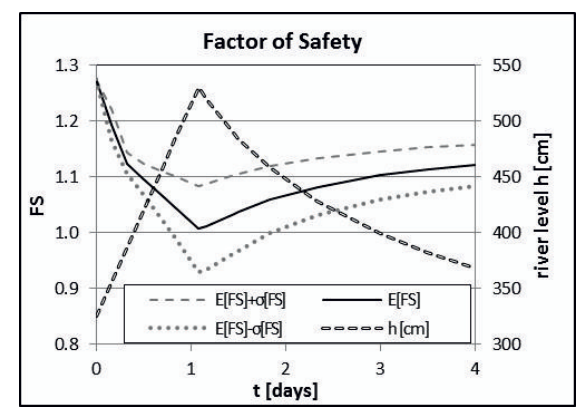

Figure 6. Factor of safety versus time and hydrograph.

The relative contribution of each variable to the variance of the factor of safety can be evaluated as:

$$
w\left[X_{i}\right]=\frac{\left(\frac{\partial g}{\partial X_{i}}\right)^{2} \sigma^{2}\left[X_{i}\right]}{\sigma^{2}[Y]}
$$

Results are shown in Fig. (7). The sum of all the terms $w\left[X_{i}\right]$ is equal to 1 and the contribution of each term is represented by its area. For every instant, the contribution of each term $w\left[X_{i}\right]$ is represented by the distance between the corresponding area's top and bottom boundaries. As expected, the contribution of $h_{\text {start }}$ is $100 \%$ at the initial time and becomes zero after the peak; on the other hand, $\alpha$ has a contribution, although not very relevant, only after the peak. At the peak (1.083 days), when the mean of the factor of safety reaches its minimum, more than $80 \%$ of the contribution to the variance of the factor of safety is given by the input variable $h_{\text {peak }}$. For this reason when applying the Monte Carlo method only the uncertainty in $h_{\text {peak }}$ is taken into account.

\subsection{Monte Carlo}

For the application of Monte Carlo method to stability analysis, the values of the input variables are randomly sampled from their cumulative probability distribution function in order to generate random hydrographs. Every random hydrograph thus generated represents the boundary condition for one seepage analysis; the distribution of pore water pressure derived from each seepage analysis is used to perform one stability analysis and calculate one value of the factor of safety. By repeating this process a number of times, a large number of values of the factor of safety are obtained and therefore its empirical cumulative distribution function can be derived. The empirical distribution function is fitted with a normal distribution in order to compare results with the ones obtained with FOSM method.

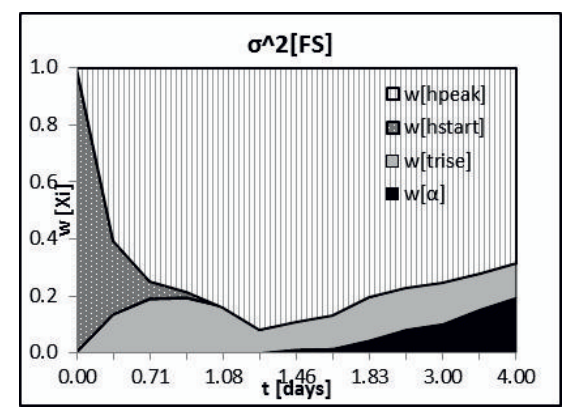

Figure 7. Relative contribution of each random variable to the variance of the Factor of Safety with respect to time.

The first step in the application of Monte Carlo method is therefore the generation of a set of random numbers between 0 and 1 ; to every random number a value of the input variable $h_{\text {peak }}$ can be associated via its cumulative probability distribution function shown in Fig. (2). The variability of $h_{\text {start }}, t_{\text {rise }}$ and $\alpha$ is neglected and these variables are kept constant and equal to their mean values for the definition of all the hydrographs, as explained in section 5.1.

The seepage analyses have been run with the software Comsol Multiphysics (Subsurface flow and porous media package). In each seepage analysis the distribution of pore water pressure on the critical slip surface has been evaluated and used as input for the stability analysis.

A routine in Matlab has been written to perform the following tasks:

1. Generate one random number $r_{i}$ between 0 and 1 ;

2. Associate $r_{i}$ to a value of $h_{\text {peak }}$ through its Gumbel cumulative probability distribution function;

3. Generate the hydrograph and apply it as boundary condition for the seepage analysis in Comsol Multiphysics;

4. Run seepage analysis in Comsol Multiphysics;

5. Extract the distribution of pore water pressure on the critical slip surface;

6. Assess the factor of safety with Bishop's simplified method;

7. Repeat steps from 1 to 6 for 400 times.

The resulting 400 values of factor of safety at the peak are plotted in Fig. (8), while the evolution in time of 
its mean value and standard deviation is plotted in Fig. (9). Comparison with FOSM results show a very good match for both plots.

The empirical cumulative probability distribution function of the factor of safety is plotted in Fig. (10). It has been fitted with a normal distribution with maximum likelihood estimation; the mean and standard deviation are reported in Table (4). They match satisfactorily the results obtained with FOSM.

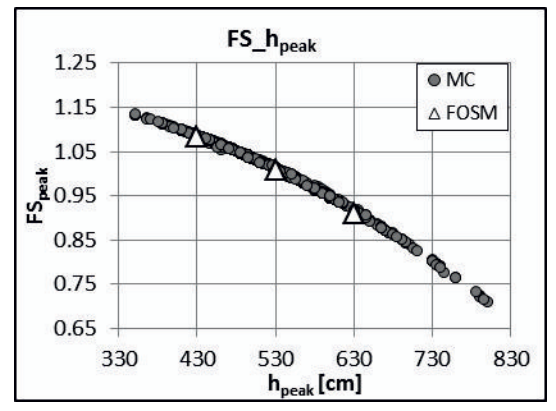

Figure 8. Factor of Safety versus river level at peak.

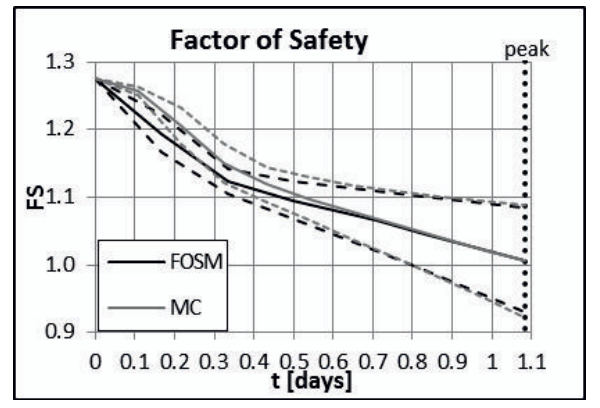

Figure 9. Factor of safety versus time obtained with FOSM and Monte Carlo methods.

Table 4. Mean and standard deviation of the Factor of Safety estimated with FOSM and Monte Carlo methods.

\begin{tabular}{|c|c|c|}
\hline \multirow{2}{*}{ FOSM } & E[FS $]$ & 1.007 \\
\cline { 2 - 3 } & $\sigma[\mathrm{FS}]$ & 0.077 \\
\hline \multirow{2}{*}{$\mathrm{MC}$} & $\mathrm{E}[\mathrm{FS}]$ & 1.006 \\
\cline { 2 - 3 } & $\sigma[\mathrm{FS}]$ & 0.083 \\
\hline
\end{tabular}

\section{Conclusions}

For the stability analysis of flood embankments it is important to take into account uncertainties in the hydraulic loading, which is represented by natural phenomena such as flood events. Results in this paper show that the variability of the maximum river level $h_{\text {peak }}$ has a significant effect on the variance of the factor of safety of the embankment and it cannot be neglected.

Probabilistic methods are a suitable approach in this case. FOSM method offers a very simple method; it has some limitations because an assumption has to be made about the probability distribution function of the factor of safety. Moreover it is not a suitable tool when the performance function has a highly non-linear behaviour.
Comparison with results obtained with Monte Carlo method show that the assumption of a normal distribution for the factor of safety is appropriate and that the factor of safety varies almost linearly with the maximum river level $h_{\text {peak }}$. The match between mean and standard deviation of the factor of safety obtained with FOSM and Monte Carlo method is very satisfactory. The number of simulations required by FOSM to obtain the same accuracy is more than 40 times less than the one required by Monte Carlo method, that can become quite heavy and time-consuming when more than one input random variable needs to be taken into account. Results show that FOSM method is a suitable and affordable approach in terms of time and number of simulations required to achieve sufficiently accurate results.

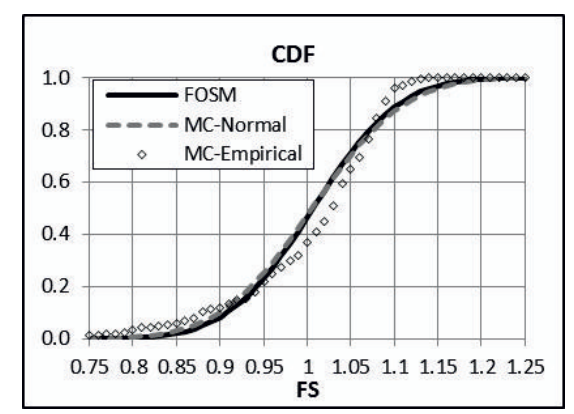

Figure 10. Cumulative distribution function of the Factor of Safety from FOSM and Monte Carlo methods.

\section{Acknowledgements}

The authors wish to acknowledge the support of the European Commission via the Marie Curie IRSES project GREAT 'Geotechnical and geological Responses to climate change: Exchanging Approaches and Technologies on a world-wide scale' (FP7-PEOPLE2013-IRSES-612665). The authors also wish to acknowledge the support of the Autonomous Province of Bolzano, Italy.

\section{References}

1. I. F. Otálvaro, M. P. Cordão-Neto, Probabilistic analyses of slope stability under infiltration conditions. Advances in Unsaturated Soils (Caicedo et al. (eds), Taylor \& Francis Group, 2013).

2. G. A. Fenton, Simulation. ALERT Doctoral School 2014 - Stochastic analysis and inverse modelling (M. A Hicks, C. Jommi (eds), 2014).

3. M. Marseguerra, E. Zio, Basics of the Monte Carlo Method with Application to System Reliability (LiLoLe-Verlag, 2002).

4. M. Th. Van Genuchten, A closed-form equation for predicting the hydraulic conductivity of unsaturated soils, Soil Science Society American Journal 44(5): 892-898 (1980). 